\title{
Multiple bottlenecks in information processing? An electrophysiological examination
}

\author{
WERNER SOMMER and HARTMUT LEUTHOLD \\ Humboldt-Universität, Berlin, Germany \\ and \\ TORSTEN SCHUBERT \\ Max-Planck-Institut für Neuropsychologische Forschung, Leipzig, Germany
}

\begin{abstract}
When two stimuli are to be processed in rapid succession, reaction time (RT) to the second stimulus is delayed. The slowing of RT has been attributed to a single processing bottleneck at response selection (RS) or to a central bottleneck following the initiation of the first response. The hypothesis of a response initiation bottleneck is mainly based on reports of underadditive interactions between stimulus onset asynchrony (SOA) and the number of stimulus-response alternatives (simple vs. two-choice response). The present study tested the hypothesis of a response initiation bottleneck by recording the lateralized readiness potential (LRP), a brain wave, emerging during or immediately following RS. The LRP findings were consistent with a central bottleneck but did not support the late bottleneck hypothesis. Instead, the LRP provided direct evidence that the underadditive interaction of number of alternatives and SOA is due to an increase of response anticipations in the simple response condition.
\end{abstract}

An important question in the study of human performance is that of the scope and limits in conducting several tasks at a time. This question has frequently been studied in the overlapping task paradigm, where separate responses $\left(\mathrm{R}_{1}\right.$ and $\left.\mathrm{R}_{2}\right)$ are required to two stimuli $\left(\mathrm{S}_{1}\right.$ and $\left.\mathrm{S}_{2}\right)$, delivered at short stimulus onset asynchronies (SOAs). Typically, reaction time to $\mathrm{S}_{1}\left(\mathrm{RT}_{1}\right)$ is stable across SOAs, whereas RT to $\mathrm{S}_{2}\left(\mathrm{RT}_{2}\right)$ is long at short SOAs and decreases as SOA is increased. This delay of $\mathrm{RT}_{2}$ at short SOAs is often explained by invoking a bottleneck stage within the information processing chain that can be occupied by only one task at a time (Welford, 1952). Whenever a bottleneck stage is required by both tasks, processing of $S_{2}$ is temporarily halted while the stage is processing $\mathrm{S}_{1}$. The halt in $\mathrm{S}_{2}$ processing causes a delay, or slack time, called psychological refractory period (PRP), which depends on the SOA. Slack is more pronounced when SOA is short because, here, the bottleneck is occupied for a longer interval relative to the arrival of $S_{2}$. Although this notion appears to be widely accepted, controversial suggestions have been made concerning the possible number of such bottlenecks and their locus within the information processing system.

Most often it is assumed that there is a central bottleneck located at the response selection stage (Pashler,

We appreciate a most inspiring discussion of this experiment with Alan Osman; helpful comments on a previous version of the manuscript by Steve Luck, Mark Van Selst, and Michael Ziessler; and the help in data collection and analysis by Susanne Großmann, Diana Mietk, Timur Kolinko, and Nele Wild-Wall. Correspondence should be addressed to W. Sommer, Institut für Psychologie, Humboldt-Universität zu Berlin, Hausvogteiplatz 5-7, 10117 Berlin, Germany (e-mail: werner.sommer@ rz.hu-berlin.de).
1994; Welford, 1980; see Figure 1). Arguments in favor of a central bottleneck involve the interaction between SOA and experimental factors that influence $\mathrm{RT}_{2}$. As Pashler and Johnston (1989) have outlined, any experimental factor that affects the time demand for processing $\mathrm{S}_{2}$ during the bottleneck or in subsequent stages will alter $\mathrm{RT}_{2}$ by a constant amount, independent of SOA. That is, although total processing time from $\mathrm{S}_{2}$ to $\mathrm{R}_{2}$ effectively shortens as increasing SOA diminishes the slack preceding the bottleneck, any manipulations within or after the bottleneck stage will merely add up to the effects of changes in SOA. Conversely, if an additive effect is found between SOA and a factor with known location of action, it can be used to localize the bottleneck. Additive effects with SOA have been reported, for example, for stimulusresponse compatibility and stimulus repetition (McCann \& Johnston, 1992; Pashler \& Johnston, 1989). Because these factors are assumed to alter processing demands at response selection, it has been concluded that slack precedes response selection.

In contrast, variations in time demands in stages preceding the bottleneck may be absorbed by the slack (Pashler \& Johnston, 1989). As can be seen in Figure 1, slack, and hence the potential for absorption, is more pronounced at short than at long SOAs. Therefore, experimental factors affecting pre-bottleneck stages will have smaller effects on $\mathrm{RT}_{2}$ when SOA is short than when SOA is long. Conversely, such underadditive interactions with SOA-that is, diminishing effects of a factor with decreasing SOAindicate that the bottleneck is situated after the stage(s) that are affected by the experimental manipulation. Consistent with the idea of a response selection bottleneck, the perceptual factor stimulus intensity shows underad- 


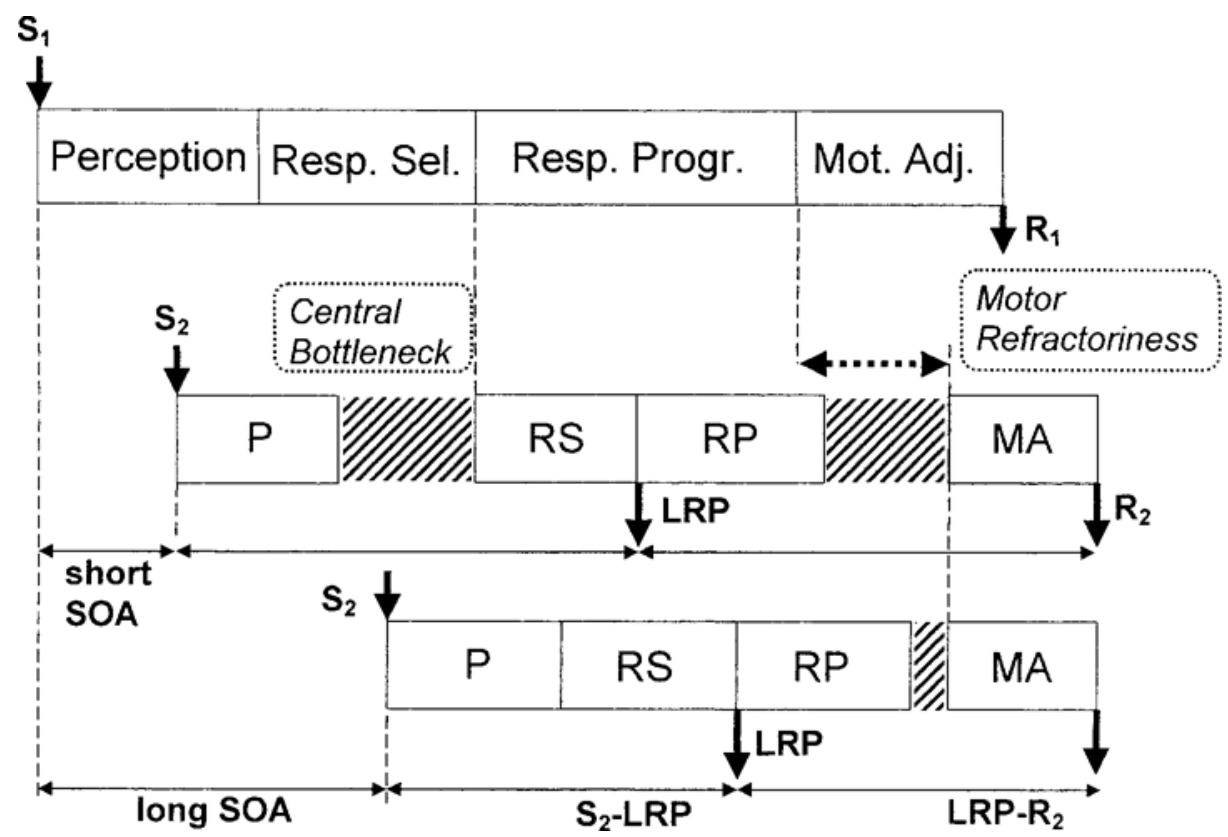

Figure 1. Model of information processing during overlapping tasks involving perception (P), response selection (RS), response programming (RP), and motor adjustment (MA). Processing from Stimulus $1\left(S_{1}\right)$ to Response $1\left(R_{1}\right)$ occurs in an uninterrupted stream. In contrast, $S_{2}-R_{2}$ processing may be interrupted by bottlenecks occurring at $\mathrm{RS}$ or due to refractoriness after the initiation of $R_{1}$. The figure illustrates how increasing stimulus onset asynchrony (SOA) may cause decreasing slacks (hatched areas) at both a central and a late motoric bottleneck. Given that the lateralized readiness potential (LRP) is generated between the two hypothetical bottlenecks, the figure also shows how the $S_{2}$-to-LRP onset and the LRP onset-to- $R_{2}$ intervals are affected by diminishing slacks at the bottlenecks. Decreasing slack at the central bottleneck decreases the $S_{2}-L R P$ interval. Importantly for present purposes, if increasing SOA diminishes slack at a late motoric bottleneck, the LRP- $\mathbf{R}_{2}$ interval should be shortened.

ditive interactions with SOA (see, e.g., De Jong, 1993; Pashler \& Johnston, 1989).

A different suggestion for the location of a bottleneck within the information processing system can be traced to a report by Karlin and Kestenbaum (1968). In some of their conditions, they manipulated the number of alternatives in the second task by requiring participants to make either simple responses or two-choice responses to $\mathrm{S}_{2}$. As a result they found that the RT difference between the simple response task and the two-choice response task was smaller for short than for long SOAs, yielding an underadditive interaction of SOA and number of alternatives on $\mathrm{RT}_{2}$. According to some authors (e.g., Donders, 1868; Fletcher \& Rabbitt, 1978; Sternberg, 1969), the effect of manipulating the number of response alternatives is assumed to be localized mainly at the stage of response selection. The results of Karlin and Kestenbaum led Keele (1973) to suggest that the bottleneck is not localized at the response selection but at late motoric stages. He suggested that after the initiation of the first response $\left(\mathrm{R}_{1}\right)$, motoric stages are temporarily unable to initiate the second response $\left(R_{2}\right)$. This causes a slack that may ab- sorb part of the increased time demand for the response selection stage in the two-choice response relative to the simple response condition. Absorption of the number of alternatives manipulation is more pronounced at short SOAs where slack is maximal than at long SOAs. Therefore the difference in $\mathrm{RT}_{2}$ between the two-choice and simple response conditions is smaller at short than at long SOAs, resulting in the observed underadditive interaction of SOA and number of alternatives.

To resolve the discrepancy between the assumption of a bottleneck localized at the stage of response selection and a bottleneck localized at later motoric stages, De Jong (1993) suggested a hybrid model, illustrated in Figure 1. This model accommodated the controversial positions by combining a central response selection bottleneck and a late response initiation bottleneck. De Jong suggested that at long intervals between $R_{1}$ and $R_{2}$ - the interresponse interval (IRI) - the motor stage that had been temporarily refractory after $R_{1}$ is able to initiate $R_{2}$ without any delays. In this case only a central bottleneck is effective, causing $\mathrm{RT}_{2}$ delays at short SOAs. However, when the IRI is small, the initiation and execution of $R_{2}$ is post- 
poned because of motor refractoriness, in effect causing a bottleneck at motoric processing stages in addition to the central bottleneck.

Recently, the interpretation of Karlin and Kestenbaum's (1968) results in terms of a late bottleneck was challenged by Schubert $(1996,1999)$ and Van Selst and Jolicœur (1997). Although they confirmed the underadditivity of SOA and number of alternatives for $S_{2}$ when simple responses were compared with two-choice responses, the additional increase of $\mathrm{RT}_{2}$ when three- or four-choice responses were introduced was independent of SOA; that is, number of alternatives was additive with SOA. The latter observation is incompatible with the idea of a bottleneck following response selection unless one assumes that in the two- to four-choice conditions, processing duration in the $S_{2}-R_{2}$ cycle relative to the $S_{1}-R_{1}$ cycle is too long to allow any slack at response-related stages.

Schubert (1996, 1999) and Van Selst and Jolicœur (1997) suggested that the underadditive interaction of SOA and number of alternatives (simple vs. two-choice responses) may be due at least partially to the unique opportunity for stimulus anticipation and early response preparation provided by the simple response condition. In the simple response condition the response is known in advance. Therefore, participants may prepare for its execution prior to stimulus delivery. Because the preparation of a response requires some minimum amount of time, preparation will be better for longer SOAs, allowing for increasingly shorter $\mathrm{RT}_{2} \mathrm{~s}$. The possibility of better preparation at long SOAs relates to peculiarities of the usual experimental procedure in PRP tasks: The stimuli are often presented with equal likelihood at each SOA. Therefore, the conditional probability for the delivery of $S_{2}$ will increase across SOAs to $100 \%$ for the longest SOA, enabling the participants to anticipate the moment of $S_{2}$ presentation and execute $R_{2}$ with a minimum or no processing of $S_{2}$. The increasing likelihood of highly prepared and anticipated responses across SOA may cause the disproportional shortening of $\mathrm{RT}_{2} \mathrm{~s}$ in the simple relative to the two-choice condition. In the twochoice response condition such anticipations are much less likely because the response is not known in advance. Similar arguments have been put forth by Frith and Done (1986) in order to explain the differences in processing speed between simple and choice reaction tasks. Schubert $(1996,1999)$ and Van Selst and Jolicœur (1997) provided evidence that makes the contributions of anticipations to the underadditive interaction plausible. Unfortunately, this evidence is indirect, being based, for example, on a relatively high number of very short RTs at long SOAs interpreted as anticipatory reactions. On the other hand, even if anticipations do contribute to the underadditive interaction, this would not rule out the presence of a late bottleneck. Anticipations and a late bottleneck may easily coexist.

One way to obtain more direct evidence for processing bottlenecks and response anticipations in overlapping tasks is to supplement performance measures with recordings of the lateralized readiness potential (LRP). The LRP is extracted from event-related brain potentials (ERP), recorded above the motor cortices. In tasks that call for left- and right-hand responses, the ERP above the cortex ipsilateral to the hand required in a given trial is subtracted from the contralateral ERP. When these difference waves are averaged they yield the LRP, reflecting pure hand-related ERP asymmetry. The LRP is considered a measure of response activation or preparation at the level of the cerebral motor cortex (see Coles, 1989; Miller \& Hackley, 1992). The interval between a stimulus and the onset of the stimulus-synchronized LRP ( $\mathrm{S}-\mathrm{LRP}$ interval) is a relative measure for the duration of premotoric processes. The S-LRP interval is sensitive, for example, to stimulus quality (Smulders, Kenemans, \& Kok, 1996) and symbolic S-R compatibility (Smulders, 1993; Smulders et al., 1996). On the other hand, the interval between the onset of the response-synchronized LRP and the response (LRP-R interval) is a relative measure for the duration of motoric processes and is influenced by response complexity (Smulders et al., 1996), by partial advance information about a forthcoming response alternative (Leuthold, Sommer, \& Ulrich, 1996), and by instructions to postpone the selected response (Sommer, Leuthold, Abdel-Rahman, \& Pfütze, 1997). Furthermore, Miller and Ulrich (1998) have shown that when the response hand can be selected, an LRP will emerge even if other aspects of response selection (e.g., selection of response finger) have yet to be completed. The findings strongly indicate that the LRP is generated prior to stages related to response initiation. It even appears safe to say that the LRP emerges either during or immediately after response selection.

Osman and Moore (1993) have demonstrated that in overlapping task situations, independent LRPs to $S_{1}$ and $S_{2}$ (and also $R_{1}$ and $R_{2}$ ) can be recorded, given that $S_{1}$ and $S_{2}$ both require left- and right-sided responses that are independent of each other. Osman and Moore reported that the interval between $\mathrm{S}_{2}$ and LRP onset decreases as SOA increases. In contrast, the interval between LRP onset and $\mathrm{R}_{2}$ is unaffected by SOA. These findings were taken to indicate that the LRP is generated during or after the bottleneck stage. According to the putative source of the LRP at or immediately after response selection, this speaks against a bottleneck at later processing stages; one has to keep in mind, however, that the Osman and Moore study was not designed to induce motor refractoriness by inducing conditions with short IRIs.

The LRP can be used to assess the existence of a late response initiation bottleneck and to more precisely specify the nature of such a bottleneck. Processing between response selection and the overt response is taken to consist of several subprocesses (e.g., Sanders, 1998). At least a final transition stage from central to peripheral motor activity (motor adjustment) has to be distinguished from a preceding motor programming stage. Unfortunately, the proponents of a late response initiation bottleneck do 
not specify whether this bottleneck is located prior to or after motor programming.

From the available evidence, it is reasonably clear that the LRP emerges before motor adjustment. If the late bottleneck is located immediately before motor adjustment, it should have specific effects on the LRP- $R_{2}$ interval, as illustrated in Figure 1. To the degree that such a bottleneck becomes effective and induces slack for $\mathrm{R}_{2}$ initiation, as should be the case for short SOAs, the LRP- $\mathrm{R}_{2}$ interval should increase relative to a situation without slack. Slack preceding $R_{2}$ initiation should be larger for short than for long SOAs. Therefore, the LRP- $\mathrm{R}_{2}$ interval should decrease with increasing SOA according to the hypothesis of a response initiation bottleneck. Slack should also be larger for short than for long intervals between $R_{1}$ and $R_{2}$ because short IRIs should be determined more by motor refractoriness than should long IRIs. Therefore, the hypothesis of a response initiation bottleneck also predicts that the $\mathrm{LRP}-\mathrm{R}_{2}$ interval should decrease with increasing IRIs. In contrast, if the response initiation bottleneck is located after response selection but precedes the LRP-generating stage(s), or if there is no such bottleneck, the LRP- $\mathrm{R}_{2}$ interval should be constant across SOAs and IRIs.

Alternatively, the anticipation hypothesis (Schubert, 1999; Van Selst \& Jolicœur, 1997) suggests that the underadditivity of SOA with number of alternatives is caused by a preponderance of response anticipations in the simple response condition relative to the two-choice response condition, especially at long SOAs. If this is true, we might expect an LRP activation in advance of $\mathrm{S}_{2}$ for simple responses but not for two-choice responses. In addition, anticipatory LRP activation, as reflected in the $\mathrm{S}_{2}$-LRP interval for simple responses, should be more pronounced at long than at short SOAs.

In sum, the present experiment was designed to assess two competing explanations for the underadditive interaction reported between SOA and the number of alternatives. Whereas the late bottleneck hypothesis predicts decreasing LRP onset-to-response intervals with increasing SOA, according to the anticipation hypothesis there should be very early onsets of the LRP when $S_{2}$ requires a simple response.

\section{METHOD}

Sixteen right-handed participants ( 9 women) contributed data to this experiment. Participants reported normal hearing and normal or corrected-to-normal vision.

Auditory stimuli were 60-dB sinusoidal tones of 1000 and $1075 \mathrm{~Hz}$ and 60-msec duration presented via loudspeaker in front of the participant. Visual stimuli were the letters X or O shown at the center of a monitor for $200 \mathrm{msec}$. All visual stimuli were presented in the midsaggital plane of the participants slightly below eye level at a fixed distance of $1 \mathrm{~m}$, provided by a chinrest. Responses were recorded with four adjacent response keys, assigned to the index and middle fingers of the left and right hands.

Each trial began with the presentation of a fixation cross followed after $500 \mathrm{msec}$ by a tone $\left(\mathrm{S}_{1}\right)$, followed in turn by one of the letters $\left(S_{2}\right)$. SOAs between tone and letter were equiprobably 100 ,
400 , or $700 \mathrm{msec}$. The two-choice responses to the tones $\left(\mathrm{S}_{1}-\mathrm{R}_{1}\right)$ were performed with the index or middle fingers of left and right hand, with fingers being counterbalanced over participants. In the two-choice response condition for $\mathrm{S}_{2}-\mathrm{R}_{2}$, the two letters required choice responses with the two other fingers. For example, if tones required choice responses with left and right index fingers, letters were to be responded to with the middle fingers. In the simple response condition, both letters required a response with just one of these fingers, alternating blockwise between left and right hand. Participants were to respond as quickly as possible to each of the two stimuli but to give priority to $S_{1}$. At the beginning of each trial block, a message on the screen indicated whether left- or right-hand simple responses or two-choice responses were required to $\mathrm{S}_{2}$. The simple response and two-choice response conditions were conducted in consecutive halves of the recording session, counterbalanced in order across participants. The recording session was conducted 1 to 3 days after an identical practice session.

Continuous recordings were made with Sn electrodes of the electroencephalogram from the positions $C^{\prime} 3$ and $C^{\prime} 4$ (Coles, 1989) with the right mastoid as common reference and of the horizontal and vertical electrooculograms (bipolar recordings) from the outer canthi and from above and below the right eye, respectively. Offline, the continuous records were digitally filtered with a bandpass of $0.01-10 \mathrm{~Hz}$ and separated into epochs of $3 \mathrm{sec}$ centered around the stimuli or responses. Difference waves were calculated between contralateral and ipsilateral recording sites. Correct and artifact-free trials were then averaged separately for left- and right-sided fingers, stimuli $\left(\mathrm{S}_{1}, \mathrm{~S}_{2}\right)$, and conditions (two-choice responses or simple responses to $S_{2}$ ). Finally, the averaged difference waves were also averaged across left and right response sides. Note that alternating blocks of simple responses between the left and right hands also allowed us to record LRPs in this condition. The onset latencies of stimulus- and response-synchronized LRPs (Table 1) ${ }^{1}$ were measured as the point in time where $50 \%$ of the peak amplitude was reached. Onsets were statistically assessed with the jackknifing procedure suggested by Miller, Patterson, and Ulrich (1998), which allows pair-wise comparisons between conditions of interest.

\section{RESULTS AND DISCUSSION}

Overall, the performance pattern in the present experiment was as expected. The upper part of Figure 2 shows the RT results. As typically observed in overlapping task situations, $\mathrm{RT}_{2}$ decreased as $\mathrm{SOA}$ increased, which reflects the existence of a processing bottleneck. Furthermore, in line with expectations, $\mathrm{RT}_{1}$ was relatively stable across SOAs. The manipulation of the number of alternatives in the second task had two main effects: First, the general level of both $\mathrm{RT}_{1}$ and $\mathrm{RT}_{2}$ appeared to be lower for the simple RT than for the two-choice condition. More importantly for the present study, the decrease of $\mathrm{RT}_{2}$ with increasing SOA was more pronounced in the simple than in the two-choice condition, replicating the findings of Karlin and Kestenbaum (1968), Schubert (1999), and Van Selst and Jolicœur (1997). Note that this result reflects the underadditive interaction of SOA and number of alternatives, interpreted by various authors (e.g., De Jong, 1993; Karlin \& Kestenbaum, 1968; Keele, 1973; Meyer \& Kieras, 1997) as evidence for a late- that is, postresponse selection-bottleneck.

As was expected, an analysis of variance (ANOVA) with repeated measures on experimental condition (simple response, two-choice response) and SOA (100, 400, 

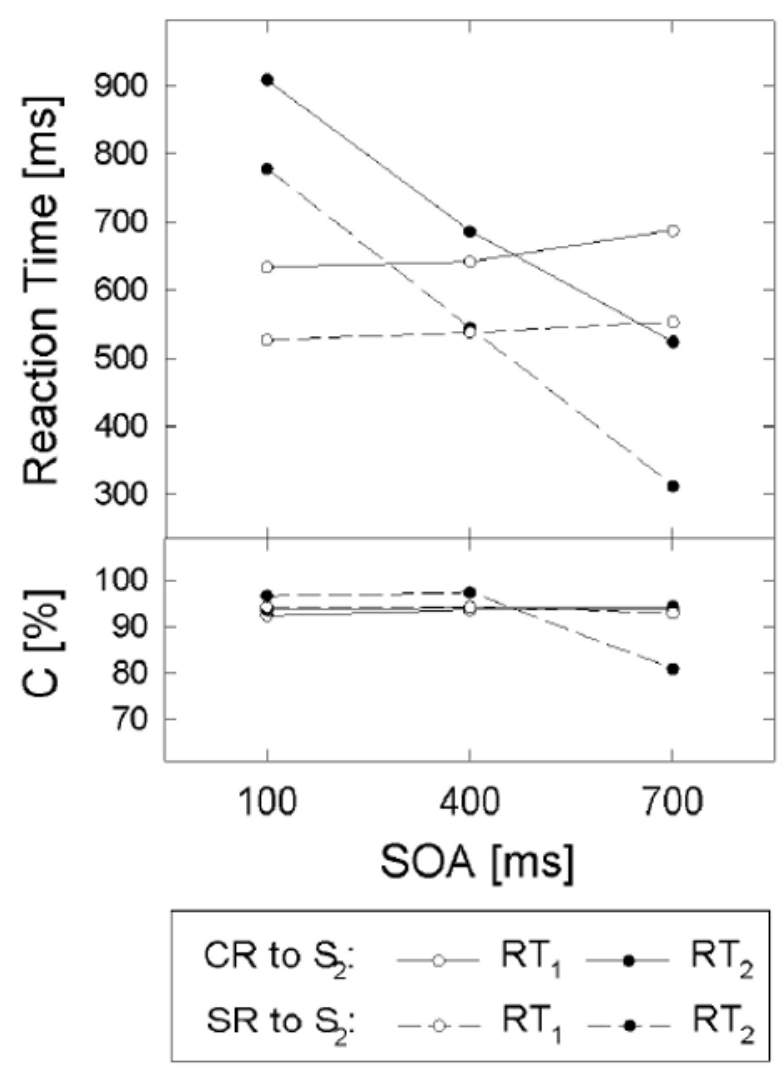

Figure 2. Reaction times (RTs) and percentage of correct responses (C[\%]) for $S_{1}$ and $S_{2}$ when either simple responses (SRs) or two-choice responses (CRs) were required to $S_{2}$.

$700 \mathrm{msec}$ ) showed the decrease of $\mathrm{RT}_{2}$ with $\mathrm{SOA}$ to be significant $[F(2,30)=5.15, \varepsilon=0.64, p<.001]$. In addition, there was a main effect of condition $[F(1,15)=$ $33.2, p<.001]$. More importantly, the disproportional shortening of $\mathrm{RT}_{2}$ with SOA in the simple response condition was also significant $[F(2,30)=13.2, \varepsilon=.68, p<$ $.001]$. Obviously this interaction between condition and SOA was mainly caused by a larger difference between $\mathrm{RT}_{2}$ for two-choice response and simple response conditions for SOA $700(M=211.7 \mathrm{msec})$ than for SOAs 100 and $400(M \mathrm{~s}=130.5$ and $140.0 \mathrm{msec}$, respectively $)$ : When the SOA 700 was omitted from the ANOVA, the SOA $\times$ condition interaction vanished $(F=1)$.

ANOVA of $\mathrm{RT}_{1}$ indicated a main effect of experimental condition $[F(1,15)=35.6, p<.001]$, reflecting the 115 -msec increase of $\mathrm{RT}_{1}$ when two-choice responses rather than simple response were required to $\mathrm{S}_{2}$. However, there was also a trend for an increase of $\mathrm{RT}_{1}$ across SOAs $[M=580,590$, and $620 \mathrm{msec}, F(2,30)=4.11$, $\varepsilon=.59, p=.05]$

The lower part of Figure 2 shows the percentage of correct responses for the various experimental conditions, incorrect key presses and responses occurring prior to the stimulus were scored as errors. Obviously there was only one condition where accuracy was relatively low: simple responses to $S_{2}$ at SOA 700. This impression was confirmed by an ANOVA with repeated measures on experimental condition, $\mathrm{SOA}$, and stimulus $\left(\mathrm{S}_{1}, \mathrm{~S}_{2}\right)$, yielding a three-way interaction $[F(2,30)=7.99, \varepsilon=0.53$, $p<.05]$; this interaction vanished when SOA 700 was omitted from the ANOVA $(p=.18)$.

Although there was little effect of SOA on $\mathrm{RT}_{1}$, we also assessed the LRP in the $S_{1}-R_{1}$ cycle. Neither the $\mathrm{S}_{1}$-LRP nor the LRP- $\mathrm{R}_{1}$ interval differed as a function of SOA $[-0.66<t<0.26]$, replicating similar findings by Osman and Moore (1993) and confirming that $S_{1}$ processing is unaffected by $S_{2}$ processing in either task/condition.

Figure 3 shows the LRP waveshapes for the $S_{2}-R_{2}$ cycle. In both experimental conditions, the $S_{2}$-LRP interval decreased with increasing SOA (see also Table 1). When two-choice responses were required, the $\mathrm{S}_{2}-\mathrm{LRP}$ interval decreased on average by $145 \mathrm{msec}$ from SOA 100 to SOA $400[t(15)=-3.24, p<.01]$ and by $100 \mathrm{msec}$ from SOA 400 to SOA $700[t(15)=-5.92, p<.01]$. In the simple response condition, the $\mathrm{S}_{2}-\mathrm{LRP}$ interval decreased from SOA 100 to SOA 400 on average by $265 \mathrm{msec}[t(15)=-5.63, p<.01]$ and by $240 \mathrm{msec}$ from SOA 400 to SOA $700[t(15)=-4.86, p<.01]$. Thus, in the longest SOA the LRP started even before $\mathrm{S}_{2}$ was presented.

The effects of SOA on the $\mathrm{S}_{2}-\mathrm{LRP}$ interval in the twochoice response condition were to be expected from a central bottleneck that precedes the elicitation of the LRP; these effects also replicate the findings of Osman and Moore (1993) and Sommer et al. (1997). As SOA shortens, slack increases and so does the interval before the LRP emerges.

Whereas in the two-choice response condition the $\mathrm{S}_{2}$-LRP followed $\mathrm{S}_{2}$ by at least $200 \mathrm{msec}$, the simple response condition clearly showed correct LRP activation prior to $S_{2}$ at SOA 700 and very soon after the stimulus for SOA 400. Therefore, in the simple response condition participants seem to have anticipated and prepared the response to the forthcoming $\mathrm{S}_{2}$, especially at long SOAs. This suggestion is also supported by the specific increase of errors in this condition.

The early start of the LRP in the simple response condition and its shifts toward even earlier onsets as SOA increases provides direct evidence for the idea that the

Table 1

Stimulus-to-LRP Onset (S-LRP) and

LRP Onset-to-Response (LRP-R) Intervals (in Milliseconds) for Choice and Simple Response Conditions in Task 2

\begin{tabular}{ccccc}
\hline \multirow{5}{*}{ SOA } & Choice & Simple & & \multicolumn{2}{c}{ LRP-R } \\
\cline { 2 - 3 } 100 & 310 & $\mathrm{~S}_{1}-\mathrm{R}_{1}$ Cycle & & Simple \\
400 & 320 & 275 & -185 & -175 \\
700 & 325 & 280 & -200 & -170 \\
& & 280 & -210 & -180 \\
100 & 530 & $\mathrm{~S}_{2}-\mathrm{R}_{2}$ Cycle & \\
400 & 395 & 490 & -155 & -145 \\
700 & 295 & 225 & -135 & -155 \\
\hline
\end{tabular}

Note-LRP, lateralized readiness potential; S, stimulus; R, response; SOA, stimulus onset asynchrony. 


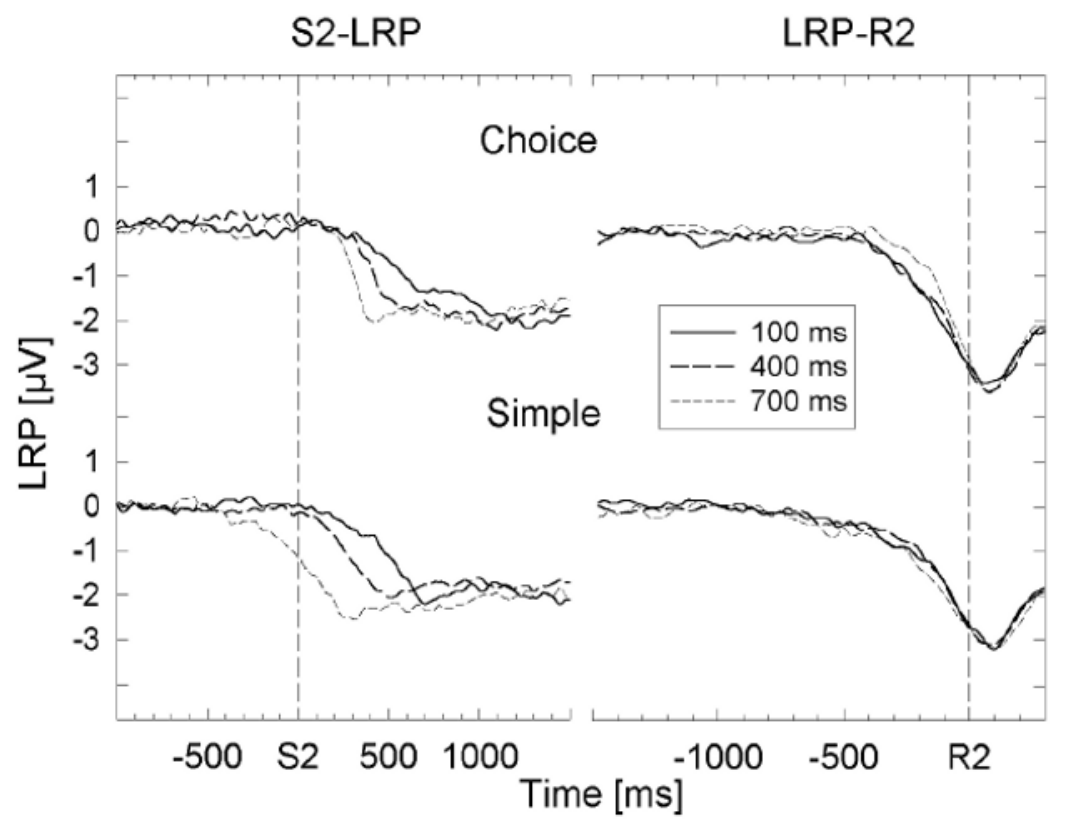

Figure 3. Stimulus- and response-synchronized lateralized readiness potentials (LRPs) in the $S_{2}-R_{2}$ cycle for two-choice and simple response tasks, superimposed for the stimulus onset asynchronies (SOAs) 100, 400, and $700 \mathrm{msec}$.

underadditivity of SOA and number of response alternatives relates to response anticipations in the simple response situation (Schubert, 1999; Van Selst \& Jolicœur, 1997). In the simple response condition there is no ambiguity about the required response, and, as SOA increases, temporal uncertainty decreases, reaching a minimum at the longest SOA, and finally there is increasing time to prepare the response. All these conditions are highly favorable for response anticipations. Thus at long SOA participants probably activate the motor response early but withhold the execution of the response until the stimulus is presented. This enables a fast emission of the response upon stimulus presentation and leads to an additional acceleration of $\mathrm{RT}_{2}$ in the simple response condition at long SOAs (Schubert, 1999). Since this acceleration is not possible in the two-choice response condition, the observed underadditive interaction of SOA and number of alternatives emerges.

The likelihood of anticipations notwithstanding, a late bottleneck after motor programming may still be active under some conditions. In this case its effects should be observable in the LRP- $\mathrm{R}_{2}$ interval. For one, a late bottleneck should cause a decrease of the LRP- $\mathrm{R}_{2}$ interval with increasing SOA. In the simple response task the LRP- $\mathrm{R}_{2}$ interval increased across SOAs, yielding a significant difference of $30 \mathrm{msec}$ when SOA 100 was compared with SOA $700[t(15)=-3.37, p<.01]$. Although significant, this increase of LRP- $\mathrm{R}_{2}$ intervals across SOAs is opposite in direction to what the late motoric bottleneck hypothesis predicts.

Most probably, in the present experiment the LRP- $\mathrm{R}_{2}$ for the simple response task was also affected by re- sponse anticipations. As noted, responses activated prior to $\mathrm{S}_{2}$ but executed after its presentation must have been postponed to some extent. Previous studies have shown that during the postponement of activated responses an LRP is present (e.g., Ilan \& Miller, 1999; Miller \& U1rich, 1998; Sommer et al., 1997). Because the postponement interval increases when the response is activated earlier, the LRP- $\mathrm{R}_{2}$ interval increases with SOA. Therefore, the increasing tendency for response anticipations with SOA may counteract and obscure any observable effects to the contrary caused in the LRP by a late bottleneck.

Because of the possible neutralizing effects of anticipations and a late bottleneck, a further test for the response initiation bottleneck hypothesis was provided by performing a median split of the trials from the short SOAs according to the IRIs between $\mathrm{R}_{1}$ and $\mathrm{R}_{2}$. At short SOAs, anticipations should be minimal and therefore a late bottleneck should be relatively unconcealed. On the other hand, a late bottleneck is assumed to be active mainly at relatively short IRIs (see De Jong, 1993). Mean IRIs below and above the median at SOA 100 were 293 versus $401 \mathrm{msec}$, and at SOA 400 they were 349 versus $454 \mathrm{msec}$. Because these IRIs were within the range where Van Selst and Jolicœur (1997) observed an underadditive interaction, the present experiment appears to have been appropriate for observing a late bottleneck if present. At SOA 100, the LRP- $\mathrm{R}_{2}$ intervals were identical $(M \mathrm{~s}=165 \mathrm{msec})$ for short and long IRIs and for SOA 400 this interval was shorter in the short than in the long IRI condition (145 vs. $190 \mathrm{msec}$ ), which, however, did not reach significance $(p>.1)$. This result is opposite to the expected decrease in the LRP- $\mathrm{R}_{2}$ interval with increasing 
IRI if a bottleneck following motor programming had been present.

Because of the possible concealment of bottleneck effects by anticipations in the simple response condition, we considered the two-choice response condition, where anticipations are much less likely. A late bottleneck after motor programming might be effective also during twochoice responses provided that the interval between the initiations of $R_{1}$ and $R_{2}$ is sufficiently short. Because intervals between response initiations should on average be small for short SOAs, we first compared the LRP- $\mathrm{R}_{2}$ intervals for short and long SOAs in the two-choice response condition.

LRP- $\mathrm{R}_{2}$ intervals for the two-choice response condition decreased across SOAs. Relative to SOA 100, the 20 -msec decrease of the LRP- $\mathrm{R}_{2}$ interval toward SOA 400 was not significant $[t(15)=1.63, p<.1]$, whereas the 55msec decrease toward SOA 700 was significant $[t(15)=$ $2.92, p<.01]$. These results appear to be consistent with the late bottleneck hypothesis. They are at variance with the reports of Osman and Moore (1993, Experiment 2) and Sommer et al. (1997), who did not find any effects of SOA on the LRP- $\mathrm{R}_{2}$ interval; however, as noted, these experiments were not designed to expose a late bottleneck.

As we did for the simple response condition, we also partitioned the trials from the two-choice response condition according to the IRIs. Irrespective of SOA, all trials were classified according to their IRI tercil, and $\mathrm{R}_{2}$ synchronized LRPs were calculated for each tercil. Mean IRIs in the three bins were 307, 387, and $523 \mathrm{msec}$. Figure 4 displays the LRPs superimposed for short, medium, and long IRIs. Numerically, the LRP- $\mathrm{R}_{2}$ interval decreased from $135 \mathrm{msec}$ at the short IRI to $100 \mathrm{msec}$ at the middle IRI to $95 \mathrm{msec}$ at the long IRI, in line with predictions from a late bottleneck hypothesis. However, none of the statistical comparisons between these conditions even approached significance (all $t \mathrm{~s}<1$ ).

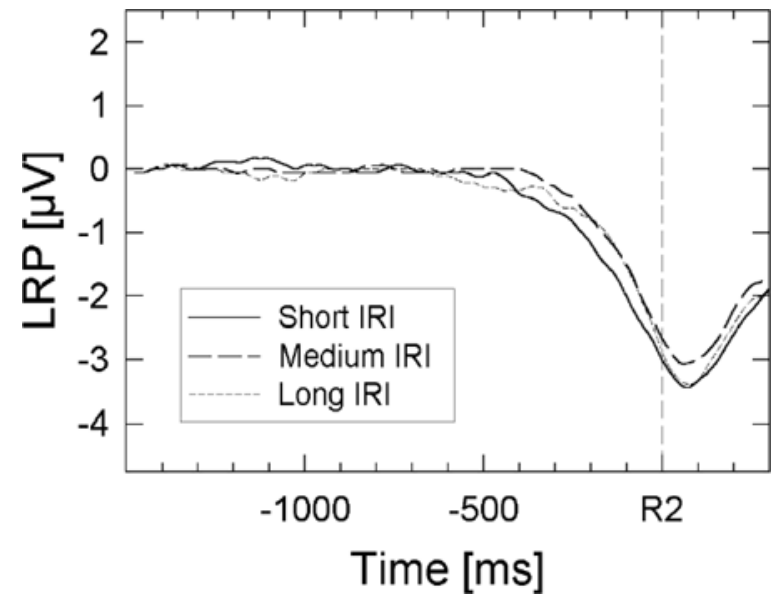

Figure 4. Response-synchronized lateralized readiness potentials (LRPs) of the two-choice response task in the $S_{2}-R_{2}$ cycle, separated for three bins of increasing interresponse intervals (IRIs).
In the present study we investigated the claim of a response initiation bottleneck, mainly based on reports of an underadditive interaction between the number of alternatives for $\mathrm{S}_{2}$ and the SOA in $\mathrm{RT}_{2}$ during overlapping task performance. We assessed this claim by recording the LRP, which we assume to be generated during or immediately after response selection but preceding the motor adjustment stage. Although we replicated the underadditive interaction in $\mathrm{RT}_{2}$ and compared conditions that should differ in the appearance of late bottleneckinduced slack, the LRP evidence for a late selection bottleneck was, at best, very slim and is far from being conclusive. Therefore we can reject with some confidence the notion that there is a bottleneck after those stages that generate the LRP. Because there is still some uncertainty whether the LRP emerges directly as a result of response selection or during response programming, we cannot completely exclude the existence of a postresponse selection bottleneck. However, if such a bottleneck following response selection exists, it would have to be located prior to the response programming stage and not immediately preceding the response adjustment stage.

On the other hand, we did find direct evidence for an alternative account of the underadditive interaction of SOA and number of alternatives in terms of response anticipations in the simple response situation (Schubert, 1996, 1999; Van Selst \& Jolicœur, 1997). The exceptional shortening of $\mathrm{RT}_{2}$ at long SOAs in the simple response condition can be explained by response anticipations. Because anticipations are less feasible in the two-choice response task, the difference between simple reaction times and choice reaction times becomes larger as SOA increases, producing the underadditive interaction of SOA and number of alternatives. The present LRP findings provide direct support for the anticipation explanation of the underadditive interaction commonly taken to indicate a late bottleneck.

\section{REFERENCES}

Coles, M. G. H. (1989). Modern mind-brain reading: Psychophysiology, physiology, and cognition. Psychophysiology, 26, 251-269.

DE JoNG, R. (1993). Multiple bottlenecks in overlapping task performance. Journal of Experimental Psychology: Human Perception \& Performance, 19, 965-980.

Donders, F. C. (1868). Over de snelheid van psychische processen: Onderzoekingen gedaan in het physiologisch laboratorium der Utrechtse Hoogeschool.[On the speed of mental processes: Research from the physiological laboratory of the University of Utrecht]. Tweede Reeks, 11, 92-120.

Fletcher, B. C., \& RABbitt, P. M. A. (1978). The changing pattern of perceptual analytic strategies and response selection with practice in a two-choice reaction time task. Quarterly Journal of Experimental Psychology, 30, 417-427.

Frith, C. D., \& Done, D. J. (1986). Routes to action in reaction time tasks. Psychological Research, 48, 169-177.

Ilan, A. B., \& Miller, J. (1999). A distinction between the initiation and the continuation of response preparation. Psychophysiology, 36, 209-219.

Karlin, L., \& Kestenbaum, R. (1968). Effects of number of alternatives on the psychological refractory period. Quarterly Journal of Experimental Psychology, 20, 167-178. 
Keele, S. W. (1973). Attention and human performance. Pacific Palisades, CA: Goodyear.

Leuthold, H., Sommer, W., \& Ulrich, R. (1996). Partial advance information and response preparation: Inferences from the lateralized readiness potential. Journal of Experimental Psychology: General, 125, 307-323.

McCann, R. S., \& Johnston, J. C. (1992). Locus of the single-channel bottleneck in dual-task interference. Journal of Experimental Psychology: Human Perception \& Performance, 18, 471-484.

Meyer, D. E., \& Kieras, D. E. (1997). A computational theory of executive cognitive processes and multiple-task performance: Part 2. Accounts of psychological refractory-period phenomena. Psychological Review, 104, 749-791.

Miller, J., \& HACKLEY, S. A. (1992). Electrophysiological evidence for temporal overlap among contingent mental processes. Journal of Experimental Psychology: General, 121, 195-209.

Miller, J., Patterson, T., \& Ulrich, R. (1998). Jackknife-based method for measuring LRP onset latency differences. Psychophysiology, 35, 99-115.

Miller, J., \& UlRich, R. (1998). Locus of the effect of the number of alternative responses: Evidence from the lateralized readiness potential. Journal of Experimental Psychology: Human Perception \& Performance, 24, 1215-1231.

Osman, A., \& Moore, C. M. (1993). The locus of dual-task interference: Psychological refractory effects on movement-related brain potentials. Journal of Experimental Psychology: Human Perception \& Performance, 19, 1292-1312.

PASHLER, H. (1994). Dual-task interference in simple tasks: Data and theory. Psychological Bulletin, 116, 220-244.

PAshler, H., \& Johnston, J. C. (1989). Chronometric evidence for central postponement in temporally overlapping tasks. Quarterly Journal of Experimental Psychology, 41A, 19-45.

SANDERS, A. F. (1998). Elements of human performance: Reaction processes and attention in human skill. Mahwah, NJ: Erlbaum.

SCHUBERT, T. (1996). Die Analyse von Interferenzeffekten bei der gleichzeitigen Bearbeitung zweier Aufgaben [The analysis of interference in overlapping tasks]. Zeitschrift für Psychologie, 4, 625-656.
SCHUbert, T. (1999). Processing differences between simple and choice reactions affect bottleneck localization in overlapping tasks. Journal of Experimental Psychology: Human Perception \& Performance, 25, 408-425.

SMULDERS, F. T. Y. (1993). The selectivity of age effects on information processing: Response times and electrophysiology. Amsterdam: University of Amsterdam Press.

Smulders, F. T. Y., Kenemans, J. L., \& KoK, A. (1996). Effects of task variables on measures of the mean onset latency of LRP depend on the scoring method. Psychophysiology, 33, 194-205.

Sommer, W., Leuthold, H., Abdel-Rahman, R., \& Pfütze, E.-M. (1997). Die Lokalisierung des Gruppierungseffektes bei überlappenden Aufgaben [The localization of the grouping effect in overlapping tasks]. Zeitschrift für Experimentelle Psychologie, 44, 103-117.

STERnBERG, S. (1969). The discovery of processing stages: Extensions of Donders' method. Acta Psychologica, 30, 276-315.

VAn Selst, M., \& Joliceur, P. (1997). Decision and response in dualtask interference. Cognitive Psychology, 33, 266-307.

WELFORD, A. T. (1952). The "psychological refractory period" and the timing of high speed performance: A review and a theory. British Journal of Psychology, 43, 2-19.

Welford, A. T. (1980). The single-channel hypothesis. In A. T. Welford (Ed.), Reaction times (pp. 215-252). London: Academic Press.

\section{NOTE}

1. Please note that LRP onsets and RTs cannot be compared directly because LRP onsets are determined much more by the extremes of the sampling distribution than are RTs. Thus, stimulus-synchronized LRP onset latencies are determined to a much larger part by the trials with early than with late onsets and response-synchronized LRP onsets are dominated by slow trials. These caveats, however, do not concern comparisons within one and the same type of dependent variable as made in the present study.

(Manuscript received August 10, 1999; revision accepted for publication March 13, 2000.) 